> Cette revue de la littérature a pour objectif de présenter le point de vue de patientes atteintes d'un cancer du sein vis-à-vis de l'utilisation des profils d'expression génique de la tumeur pour définir une stratégie thérapeutique. Nous identifions les idées, les attentes ou les craintes qui pourraient constituer une entrave, ou une facilitation, à la mise en œuvre et à la diffusion de ces méthodes en pratique clinique. Globalement, les patientes s'avèrent très favorables à l'utilisation d'un test génomique pour sélectionner le traitement le plus approprié, dans la logique du concept de médecine personnalisée. Un certain nombre de confusions et d'inquiétudes sont néanmoins observées. Leur connaissance et leur clarification ultérieure par les cliniciens devraient permettre aux patientes de prendre une part plus active et plus éclairée aux prises de décision thérapeutique, de mieux vivre la période d'annonce des traitements et d'améliorer leur vécu ultérieur. <

Le pronostic d'un cancer du sein et le bénéfice thérapeutique d'une chimiothérapie adjuvante sont généralement estimés d'après les caractéristiques histologiques et cliniques : âge de la patiente, existence d'un envahissement ganglionnaire, grade et taille de la tumeur, statut des récepteurs hormonaux et de la protéine HER2 [1]. Dans un contexte où les stratégies thérapeutiques visent à s'adapter au plus juste à chaque patient, la démarche actuelle consiste à essayer de mieux individualiser les traitements en se basant sur l'analyse des profils d'expression génétique (GEP) de la tumeur [2].

Plusieurs études ont suggéré que les GEP pouvaient être utilisés pour définir le risque de récidive après un traitement incluant ou non une chimiothérapie adjuvante, et prédire la réponse à la chimiothérapie néo-adjuvante (administrée avant la chirurgie) [3-9]. II a également été rapporté que certaines signatures

\section{Chimiothérapie ciblée du cancer du sein basée sur une analyse génomique de la tumeur}

Adhésion des patientes, craintes et malentendus
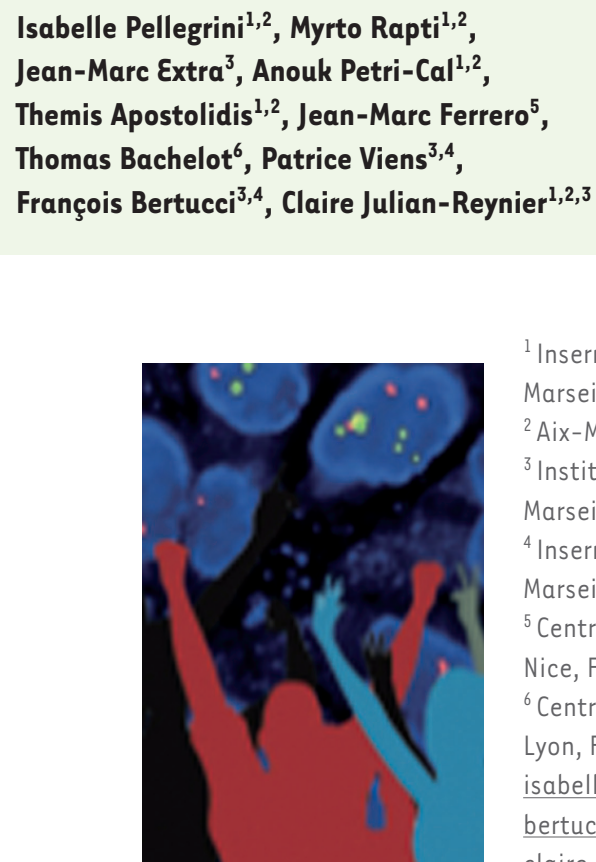

${ }^{1}$ Inserm UMR912, IRD,

Marseille, France;

${ }^{2}$ Aix-Marseille Univ, France ;

${ }^{3}$ Institut Paoli-Calmettes,

Marseille, France ;

${ }^{4}$ Inserm UMR891 CRCM,

Marseille, France ;

${ }^{5}$ Centre Antoine Lacassagne,

Nice, France;

${ }^{6}$ Centre Léon Bérard,

Lyon, France.

isabelle.pellegrini@inserm.fr bertuccif@marseille.fnclcc.fr claire.julian-reynier@inserm.fr

génomiques permettaient d'identifier les patientes les plus susceptibles de bénéficier d'une chimiothérapie adjuvante $[10,11]$.

Bien que la validité clinique des signatures génomiques soit maintenant bien établie, on commence seulement à en explorer l'utilité clinique, c'est-à-dire la façon dont elles influencent effectivement les décisions médicales et bénéficient au patient [12-14]. La majorité des médecins oncologues se déclarent très favorables à l'utilisation de ces tests comme aide à la décision thérapeutique [13, 15]. Par exemple, Lo et al. ont montré que l'utilisation en clinique du « recurrence score ${ }^{1} \gg$ conduisait les praticiens à modifier leurs recommandations thérapeutiques dans environ un tiers des cas [13].

Mais, alors que ces nouvelles procédures fondées sur la génomique pénètrent progressivement le champ clinique [16], peu de données analysent la manière dont elles sont comprises et perçues par les patients atteints de cancer [17]. Or, cette analyse des perceptions de ces nouvelles technologies par les patients atteints de cancer, et leur prise en compte dans les pratiques cliniques en oncologie, paraissent impor-

${ }^{1} 21$-gene recurrence score (RS, Oncotype $\mathrm{Dx}{ }^{\circledR}$ ). 
tantes. Une étude préliminaire a évalué l'intérêt des patientes pour l'utilisation d'un test génomique lors de la décision de la chimiothérapie, en les plaçant face à des scénarios hypothétiques [18]. D’autres études plus récentes, dont la plupart ont utilisé des auto-questionnaires, se sont intéressées aux réactions émotionnelles des patientes vis-à-vis de I'utilisation de deux signatures génomiques (Amsterdam² ${ }^{2}$ et recurrence score), à leur satisfaction, ainsi qu'à leur niveau de connaissance de ces nouvelles procédures $[13,15,19,20]$. Dans une étude qualitative fondée sur des entretiens, nous avons pu analyser en détail l'expérience de femmes atteintes d'un cancer du sein auxquelles un test génomique prédictif de la réponse à la chimiothérapie néo-adjuvante avait été proposé pour guider le choix de la chimiothérapie [21].

L'objectif de cet article est, à partir d'une revue de la littérature, d'analyser les perceptions et points de vue des patientes vis-à-vis de ces nouvelles technologies, afin d'identifier les idées, attentes ou craintes qui pourraient constituer une entrave, ou une facilitation, à leurs mises en œuvre et diffusion en pratique clinique.

\section{Adhésion globale à l'idée de traitements individualisés sur la base d'un test génomique de la tumeur}

Comme d'autres technologies basées sur la génétique ou la génomique, l'outil décisionnel thérapeutique que constituent les tests analysant le GEP des tumeurs est encore peu connu du public [17]. Or, la littérature montre que les attitudes profanes des patients, leurs perceptions de ces nouvelles technologies, sont importantes pour l'acceptation de ces innovations et leur intégration en clinique [22]. Dans les différentes études, la plupart des femmes interrogées reconnaissaient n'avoir jamais entendu parler de ces techniques auparavant, ou en avaient une compréhension relativement sommaire [19, 20].

Dans notre étude, les termes « génomique » ou « expression génique » suscitaient un certain nombre de malentendus et confusions [21]. La majorité des patientes se rappelaient avoir donné leur consentement, avant l'intervention chirurgicale, pour la réalisation d'une analyse de leur tumeur servant à guider le choix du protocole de chimiothérapie adjuvante, mais les mots « génomique » ou « expression génique » avaient une signification très floue, voire aucune signification, pour 17 patientes sur 37 :

«Analyse génonémique? Génomique? Non, je ne sais pas, je n’ai aucune idée de ce que cela signifie. Aucune idée, vraiment. La recherche, peut-être? Le génome? Non, cela ne m'évoque rien... » (Mme A, 60 ans.)

Toutefois, les patientes souscrivent aisément à l'idée d'un traitement adapté à chaque cas sur la base d'une analyse génomique de la tumeur [18]. Elles comprennent bien l'aide à la décision que constituent ces nouvelles procédures en matière de choix thérapeutiques [19]. De ce point de vue, l'analyse génomique est banalisée et ne se distingue pas particulièrement de l'ensemble des autres analyses réalisées en amont des traitements afin d'établir le programme personnalisé de soins. Le test génomique est alors considéré comme faisant partie de la batterie

${ }^{2}$ Amsterdam 70-gene prognostic profile (Mammaprint ${ }^{\circledR}$ ). de tests réalisés en routine pour caractériser leur tumeur et adapter les traitements en conséquence. À ce titre, il ne génère ni crainte, ni suspicion particulière [21]. Aussi les patientes se déclarent-elles rétrospectivement satisfaites du traitement reçu, considérant qu'il était «le plus adapté à leur cas particulier ». Selon que le résultat de leur analyse les classe dans le groupe nécessitant une chimiothérapie forte, ou plus légère, différents types d'arguments sont invoqués.

«D'après le test qu'ils ont fait sur ma tumeur, la chimiothérapie légère était suffisante. Et après les troisièmes $\mathrm{FEClOO}^{3}$, je me suis dit que j'avais vraiment de la chance de ne pas avoir le Taxotère, parce que je n'aurais pas supporté les effets secondaires. Alors oui, j'étais vraiment soulagée... »(Mme $\varepsilon, 34$ ans.)

À l'inverse, les patientes chez lesquelles l'analyse génomique ne modifiait pas les recommandations de la chimiothérapie rationalisaient généralement le résultat des tests génomiques en considérant qu'ils leur avaient permis de bénéficier du traitement «standard », c'està-dire celui qui est bien connu et qui marche.

«L'analyse a prouvé que de toute façon il fallait faire le Taxotère, on ne pouvait pas faire autrement, et donc je n'entrais pas dans le protocole expérimental. Et donc je me suis retrouvée dans le processus normal, habituel, ce qui est assez rassurant au final. » (Mme G, 57 ans.) Enfin, certaines patientes étaient aussi sensibles à l'idée d'avoir bénéficié d'une « nouvelle » stratégie de traitement, fruit de l'innovation médicale et de la recherche, à laquelle elles contribuaient aussi.

«Il y avait deux choses. Tout d'abord, l'avantage que cela représentait pour moi d'avoir le dernier traitement, le mieux adapté à ma tumeur. Et ensuite, j'ai pensé que cela aidait la recherche contre le cancer. Je crois que c'est très important de participer à la recherche, c'est comme cela que les traitements progressent. » (Mme F, 65 ans.)

Ainsi, même si le degré de connaissance et de compréhension de l'analyse génomique par les patientes reste relativement sommaire, leur attitude vis-à-vis de ces procédures est très positive. D'autres études montrent que les décisions de chimiothérapie qui se fondent sur une signature génomique, et qui suscitent un grand intérêt chez les patientes $[15,20]$, tendent même à diminuer les niveaux d'anxiété [13]. Aussi les notions de médecine personnalisée et de chimiothérapie individualisée pourraient-elles avoir un effet positif chez les patientes auxquelles vient d'être annoncé un cancer et qui se trouvent bien souvent en situation de détresse et de vulnérabilité psychique lors de cette annonce [23].

\footnotetext{
${ }^{3}$ Épirubicine (Farmorubicine), 5-FU, endoxan
} 


\section{Malentendus et craintes associés aux tests génomiques}

Malgré cette adhésion forte des patientes à l'utilisation de l'analyse génomique de leur tumeur pour adapter leur traitement de chimiothérapie, on discerne certaines craintes ou interprétations erronées, et celles-ci peuvent influencer l'acceptation que les patientes ont de ces méthodes. La perception qu'ont les femmes de leur état de santé et de leur pronostic vital est très influencée par la terminologie utilisée pour décrire la signature génomique, que ce soit par les médecins ou dans la brochure d'information et le feuillet donnés lors du recueil du consentement, et par l'interprétation qu'elles en font. Les mots utilisés pour parler de ces techniques s'avèrent donc avoir une grande importance, en raison du caractère sensible de la valeur pronostique de la signature génomique.

\section{Significations attribuées aux analyses}

\section{«non faites 》 et « non interprétables 》}

Le premier problème est le sens accordé aux analyses qui, pour des raisons techniques, ne sont pas faites ou ne sont pas interprétables, ce qui génère anxiété et incertitude pour l'avenir [21]. Si une signature positive est généralement reçue de façon positive, car signifiant que le pronostic de la maladie est bon, à l'inverse une mauvaise signature, ou une signature négative, est souvent interprétée comme l'indice d'un pronostic péjoratif. De façon plus surprenante, les termes «signature non faite » ou « signature non interprétable » étaient, dans notre étude, aussi connotés négativement et interprétés comme prédictifs d'une espérance de vie médiocre. Dans l'extrait d'entretien reproduit ci-dessous, le sens que la patiente attribue à sa signature «non faite » est celui d'un «mauvais pronostic vital».

"Les mots "bon" et "mauvais" sont répétés constamment tout au long des 3 pages de la brochure d'information. Pour le profane qui lit le protocole, n'entrent dans cet essai clinique que les cancers à bon pronostic. Et donc pour moi, ne pas entrer dans la cohorte signifiait que j'étais un "mauvais pronostic". Et quand on connaît le sens du mot "pronostic" en cancérologie... Ce que l'on peut en déduire aussi, c'est que les gens qui peuvent en mourir ne les intéressent pas. » (Mme C, 59 ans.)

De même, le récit de Mme $D$. est révélateur du poids que peut acquérir le résultat de l'analyse génomique, et de ce qu'il peut signifier pour sa vie, son avenir, ainsi que celui de son époux. S'entendre dire que son résultat était «non interprétable » produisait en elle un sentiment de profonde frustration et une incertitude accrue pour l'avenir.

«Apparemment cela permettait de rechercher dans le génome quel était le pronostic pour mon futur et une éventuelle récidive, et pour moi c'est important de savoir. Bon c'est dur à entendre, mais j'ai besoin de connaître la vérité, parce que cela influe sur l'espérance de vie. Donc j'aimerais savoir le temps a priori dans lequel on me classe pour pouvoir voir si je continue dans mes projets de vie, que ce soit faire des enfants, acheter un terrain, une maison, etc. » (Mme D, 38 ans.)

De façon analogue, Retel et al. rapportent l'impact émotionnel très négatif sur les patientes des situations où une incertitude existe sur le résultat du test génomique (qu'il n'ait pas pu aboutir pour des raisons techniques ou qu'il soit discordant avec les critères de décision histologiques et cliniques) [15].

\section{Confusion entre les mots « génomique 》 et « génétique 》}

Le deuxième malentendu réside dans les confusions et glissements sémantiques qui peuvent s'établir entre les mots « génomique », « génétique » et « héréditaire». Une proportion non négligeable (plus d'un quart) des femmes interrogées dans notre étude pensaient que l'analyse portait sur leurs gènes constitutifs; elles s'imaginaient alors à tort être porteuses d'une prédisposition génétique au cancer. Des taux encore plus élevés (67\%) étaient relevés dans l'étude de Richman [19]. Cette méprise, fondée sur la ressemblance entre les termes « génomique » et « génétique » et sur un glissement sémantique de « génétique » à « héréditaire », générait des craintes de transmission héréditaire d'un risque de cancer à leur descendance, et des sentiments de culpabilité, ainsi que l'exprime Mme B. ci-dessous :

«Bien que l'on ne me l'ait pas dit explicitement, je pensais que ce test était destiné à déterminer s'il y avait un risque de transmission héréditaire. Et à m'informer si ma fille allait développer elle aussi un cancer... Et en fait, je me sentirais assez coupable de le lui avoir transmis. » (Mme B, 50 ans.)

\section{Implications pour la clinique}

Dans l'ensemble, les patientes atteintes d'un cancer du sein souscrivent volontiers à l'idée d'un traitement de chimiothérapie adapté à chaque cas sur la base d'une analyse génomique de la tumeur. Cette attitude positive va de pair avec une adhésion très forte au concept de médecine personnalisée, évoqué par la plupart des femmes. Néanmoins, un certain nombre d'écueils relatifs aux termes employés pour décrire ces nouvelles technologies confirment que, dans la période critique de l'annonce de la maladie, la terminologie utilisée par les praticiens pour décrire les traitements à venir est une question sensible [24]. Les différentes études montrent comment les informations relatives à l'analyse génomique, aux caractéristiques moléculaires de la tumeur et aux modalités de la prise en charge thérapeutique qui en découlent sont comprises, et peuvent être réinterprétées ou mal interprétées. C'est le cas notamment pour les termes de génomique, de bonne ou mauvaise signature moléculaire, qui suscitent au plan subjectif affects anxieux et inquiétude. 
Étant donné les risques potentiels de malentendus portant sur des termes spécifiques (génomique, génique) ou non spécifiques (non faite, non interprétable), il semble impératif de porter une attention plus grande à la question de l'information dans ce champ. Dans le cas présent, deux points méritent d'être améliorés en pratique : (1) la différence entre analyse génétique (constitutionnelle, de prédisposition au cancer) et analyse génomique (de la tumeur, de prédiction du risque évolutif) doit être précisée. Les praticiens doivent expliquer que ces mots portent exclusivement sur les gènes de la tumeur et n'ont rien à voir avec un risque de transmission héréditaire. (2) L'annonce de résultats «non faits » ou «non interprétables » doit être faite avec précaution en raison de l'impact psychologique potentiellement délétère de ces termes. Cela peut affecter la façon dont le patient va vivre la suite de ses traitements et de sa prise en charge. II est important de préciser que ces termes indiquent que des problèmes techniques sont survenus lors de la réalisation du test, mais ne renvoient pas à la tumeur elle-même.

La reconnaissance de ces problèmes potentiels et leur clarification ultérieure par les cliniciens devraient permettre de prévenir - ou de soulager - une anxiété trop grande chez les patientes. Prendre plus de temps pour expliquer ce que ces termes impliquent, favoriser la discussion et encourager les questions des patientes devraient permettre à ces dernières de prendre une part plus active et éclairée aux décisions concernant leur traitement, ainsi que de mieux supporter la période d'annonce des traitements et leur vécu ultérieur. $\diamond$

\section{SUMMARY}

Targeted chemotherapy for breast cancer:

patients perception of the use of tumor gene profiling approaches to better adapt treatments

The purpose of this review of the literature is to document how breast cancer patients perceive the use of tumor gene profiling approaches to better adapt treatments, and to identify the features of these approaches that may impact their clinical application. In general, the use of tumor genomic analysis was perceived by patients as an approach facilitating personalized medicine and received considerable support. Nevertheless, a number of confusions and worries about these practices were also identified. Improving the quality of provider/patient communications should enable patients to play a more active part in the decision-making about their treatment. This will ensure that those who agree to their tumor gene analysis have realistic expectations and sound deductions of the final result disclosure process. $\diamond$

\section{CONFLIT D'INTÉRÊTS}

Les auteurs déclarent n'avoir aucun conflit d'intérêts concernant les données publiées dans cet article.

\section{RÉFÉRENCES}

1. EBCTCG. Effects of chemotherapy and hormonal therapy for early breast cancer on recurrence and 15-year survival: an overview of the randomised trials. Lancet $2005 ; 365: 1687-717$.

2. Sotiriou C, Pusztai L. Gene-expression signatures in breast cancer. N EnglJ Med 2009 ; 360 : $790-800$.
3. Bertucci F, Nasser V, Granjeaud S, et al. Gene expression profiles of poorprognosis primary breast cancer correlate with survival. Hum Mol Genet $2002 ; 11: 863-72$

4. Carey LA, Perou CM, Livasy CA, et al. Race, breast cancer subtypes, and survival in the Carolina breast cancer study. JAMA 2006 ; 295 : 2492-502.

5. Chang JC, Wooten EC, Tsimelzon A, et al. Gene expression profiling for the prediction of therapeutic response to docetaxel in patients with breast cancer. Lancet $2003 ; 362: 362-9$.

6. Hess KR, Anderson K, Symmans WF, et al. Pharmacogenomic predictor of sensitivity to preoperative chemotherapy with paclitaxel and fluorouracil, doxorubicin, and cyclophosphamide in breast cancer. J Clin Oncol 2006 ; 24 4236-44.

7. Perou CM, Sorlie T, Eisen MB, et al. Molecular portraits of human breast tumours. Nature $2000 ; 406: 747-52$.

8. Van't Veer LJ, Dai H, van de Vijver MJ, et al. Gene expression profiling predicts clinical outcome of breast cancer. Nature $2002 ; 415: 530-6$.

9. Van de Vijver MJ, He YD, van't Veer LJ, et al. A gene-expression signature as a predictor of survival in breast cancer. N Engl J Med 2002 ; 347 : 1999-2009.

10. Knauer M, Mook S, Rutgers $\varepsilon$ J, et al. The predictive value of the 70-gene signature for adjuvant chemotherapy in early breast cancer. Breast Cancer Res Treat $2010 ; 120: 655-61$.

11. Paik S, Tang G, Shak S, et al. Gene expression and benefit of chemotherapy in women with node-negative, estrogen receptor-positive breast cancer. J Clin Oncol 2006 ; 24 : 3726-34.

12. Bueno-de-Mesquita JM, van Harten WH, Retel VP, et al. Use of 70-gene signature to predict prognosis of patients with node-negative breast cancer: a prospective community-based feasibility study (RASTER). Lancet Oncol $2007 ; 8: 1079-87$.

13. Lo SS, Mumby PB, Norton J, et al. Prospective multicenter study of the impact of the 21-gene recurrence score assay on medical oncologist and patient adjuvant breast cancer treatment selection. J Clin Oncol, $2010 ; 28$ : 1671-6.

14. Oratz R, Paul D, Cohn AL, Sedlacek SM. Impact of a commercial reference laboratory test recurrence score on decision making in early-stage breast cancer. J Oncol Pract $2007 ; 3$ : 182-6.

15. Retel VP, Bueno-de-Mesquita JM, Hummel MJ, et al. Constructive technology assessment (CTA) as a tool in coverage with evidence development: the case of the 70 -gene prognosis signature for breast cancer diagnostics. Int J Technol Assess Health Care 2009; $25: 73-83$.

16. Sparano JA, Solin LJ. Defining the clinical utility of gene expression assays in breast cancer: the intersection of science and art in clinical decision making. J Clin Oncol $2010 ; 28: 1625-7$.

17. Nielsen LF, Moldrup C. Lay perspective on pharmacogenomics: a litterature review. Personalized Medicine $2006 ; 3$ : 311-6.

18. O'Neill SC, Brewer NT, Lillie SE, et al. Women's interest in gene expression analysis for breast cancer recurrence risk. J Clin Oncol 2007 ; 25 : 4628-34.

19. Richman AR, Tzeng JP, Carey LA, et al. Knowledge of genomic testing among early-stage breast cancer patients. Psychooncology $2011 ; 20: 28-35$.

20. Tzeng JP, Mayer D, Richman AR, et al. Women's experiences with genomic testing for breast cancer recurrence risk. Cancer $2010 ; 116: 1992-2000$.

21. Pellegrini I, Rapti M, Extra JM, et al. Tailored chemotherapy based on tumor gene expression analysis: breast cancer patients misinterpretations and positive attitudes. Eur J Cancer Care 2012 (sous presse).

22. Moldrup C. Ethical, social and legal implications of pharmacogenomics: a critical review. Community Genet $2001 ; 4$ : 204-14.

23. Turnquist DC, Harvey JH, Andersen BL. Attributions and adjustment to life-threatening illness. Br J Clin Psychol $1988 ; 27$ : 55-65.

24. Thorne S, Oliffe J, Kim-Sing C, et al. Helpful communications during the diagnostic period: an interpretive description of patient preferences. Eur J Cancer Care (Engl) $2010 ; 19$ : 746-54. 